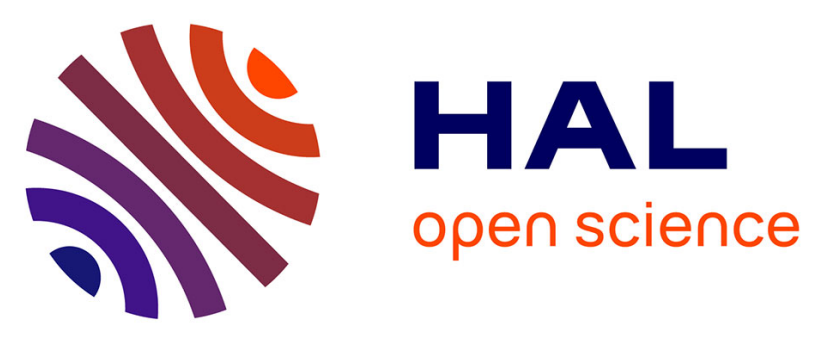

\title{
Approximating Multidimensional Subset Sum and the Minkowski Decomposition of Polygons
}

\author{
Ioannis Z. Emiris, Anna Karasoulou, Charilaos Tzovas
}

\section{To cite this version:}

Ioannis Z. Emiris, Anna Karasoulou, Charilaos Tzovas. Approximating Multidimensional Subset Sum and the Minkowski Decomposition of Polygons. Mathematics in Computer Science, 2017, 11, pp.35-48. 10.1007/s11786-017-0297-1 . hal-01401896

\section{HAL Id: hal-01401896 \\ https://hal.inria.fr/hal-01401896}

Submitted on 24 Nov 2016

HAL is a multi-disciplinary open access archive for the deposit and dissemination of scientific research documents, whether they are published or not. The documents may come from teaching and research institutions in France or abroad, or from public or private research centers.
L'archive ouverte pluridisciplinaire HAL, est destinée au dépôt et à la diffusion de documents scientifiques de niveau recherche, publiés ou non, émanant des établissements d'enseignement et de recherche français ou étrangers, des laboratoires publics ou privés.

\section{(1) (1) $\$$}

Distributed under a Creative Commons Attribution - NonCommercial - NoDerivatives 44.0 


\title{
Approximating Multidimensional Subset Sum and the Minkowski Decomposition of Polygons ${ }^{\text {th }}$
}

\author{
Ioannis Z. Emiris ${ }^{1}$, Anna Karasoulou ${ }^{1}$, Charilaos Tzovas ${ }^{1}$, \\ ${ }^{a}$ Department of Informatics $\& 3$ Telecommunications, University of Athens, Athens, Greece, \\ and "Athena" Research Center, Maroussi, Greece \\ ${ }^{b}$ Department of Mathematics, University of Athens, Athens, Greece
}

\begin{abstract}
We consider the approximation of two NP-hard problems: Minkowski Decomposition (MinkDecomp) of lattice polygons in the plane and the closely related problem of Multidimensional Subset Sum ( $k D$-SS) in arbitrary dimension. In $k D$-SS we are given an input set $S$ of $k$-dimensional vectors, a target vector $t$ and we ask, if there exists a subset of $S$ that sums to $t$. We prove, through a gap-preserving reduction, that, for general dimension $k, k D$-SS is not in APX although the classic $1 D$-SS is in PTAS. On the positive side, we present an $O\left(n^{3} / \epsilon^{2}\right)$ approximation grid based algorithm for $2 D$-SS, where $n$ is the cardinality of the set and $\epsilon$ bounds the difference of some measure of the input polygon and the sum of the output polygons. We also describe two more approximation algorithms with a better experimental ratio. Applying one of these algorithms, and a transformation from MinkDecomp to $2 D$-SS, we can approximate MinkDecomp. For an input polygon $Q$ and parameter $\epsilon$, we return two summands $A$ and $B$ such that $A+B=Q^{\prime}$ with $Q^{\prime}$ being bounded in relation to $Q$ in terms of volume, perimeter, or number of internal lattice points, an additive error linear in $\epsilon$ and up to quadratic in the diameter of $Q$. A similar function bounds the Hausdorff distance between $Q$ and $Q^{\prime}$. We offer experimental results based on our implementation.
\end{abstract}

\section{Introduction}

Every polynomial is related with its Newton polytope and, using a theorem of Ostrowski, Gao devised an irreducibility test for a polynomial. Here, we consider the problem of decomposition of integral polygons. A polygon $Q$ is called an (integral) lattice polygon, when all its vertices are points with integer coordinates.

A polygon $Q$ is called an (integral) lattice polygon, when all its vertices are points with integer coordinates.

Definition 1. The Minkowski sum of two sets of vectors $A$ and $B$ in Euclidean space is defined by adding each vector in $A$ to each vector in $B$, namely: $A+B=$

\footnotetext{
«The authors were partially supported by H2020 M. Sklodowska-Curie Innovative Training Network "ARCADES: Algebraic Representations in Computer-Aided Design for complEx Shapes", 2016-2019.

${ }^{*}$ Corresponding author.
} 
$\{a+b \mid a \in A, b \in B\}$.

Problem 2. Minkowski Decomposition (MinkDecomp).

Given a lattice convex polygon $Q$, decide if it is decomposable, that is, if there are nontrivial lattice polygons $A$ and $B$ such that $A+B=Q$, where + denotes the Minkowski sum. The polygons $A$ and $B$ are called summands.

?? is proven NP-complete in [? ] and can be reduced to $2 D$-SS. For the reduction see ??. The approximation version can be defined as follows.

\section{Problem 3. MinkDecomp- $\mu$-approx}

Input: A lattice polygon $Q$, a parameter $0<\epsilon<1$ and a function $\mu$.

Output: Lattice polygons $A, B$ such that $0 \leq \mu(A+B)-\mu(Q)<\epsilon \cdot \phi(D)$, where $D$ is the diameter of $Q$ and $\phi$ a polynomial. We call such an output an $\epsilon \cdot \phi(D)$-solution.

For $\mu$ we may consider the functions $\operatorname{vol}(Q)$ : the volume, $\operatorname{per}(Q)$ : the perimeter, $i(Q)$ : the internal lattice points of $Q$ or $d_{H}(Q, A+B)$ : the Hausdorff distance between $Q$ and $A+B$. An interesting question is what other functions we can use to measure the similarity of two polygons.

Problem 4. $k D$-Subset Sum ( $k D$-SS)

Input: A vector set $S=\left\{v_{i} \mid v_{i} \in \mathbb{Z}^{k}, 1 \leq i \leq n, k \geq 1\right\}$ and a target vector $t \in \mathbb{Z}^{k}$.

Output: Decide, whether there exists a vector subset $S^{\prime} \subseteq S$ such that $\sum v_{i}=t, v_{i} \in S^{\prime}$.

This is a generalization of the classic $1 D$-SS problem, and as such, is also NP-complete.

Let $P_{i}$ be the set of all possible vector sums that can be produced by adding at most $i$ vectors among the first vectors in $S$. Then, $P_{n} \subseteq \mathbb{Z}^{k}$ is the set of all possible vector sums. Here is the approximation version:

\section{Problem 5. $k D$-SS-opt}

Input: A set $S=\left\{v_{i} \mid v_{i} \in \mathbb{Z}^{k}, 1 \leq i \leq n, k \geq 1\right\}$, a nonzero target $t \in P_{n}$ and $0<\epsilon<1$.

Output: Find a subset $S^{\prime} \subseteq S$ whose vector sum $t^{\prime}=\sum v_{i}, v_{i} \in S^{\prime}$ that

$$
\text { minimize } \operatorname{dist}\left(t, t^{\prime}\right) \text {. }
$$

We consider the Euclidean distance $l_{2}$ here, but our method is easily generalized to any $l_{p}, 1 \leq p<\infty$. For more details see Theorem 8.22 in [? ].

Definition 6. A PTAS (Polynomial Time Approximation Scheme) is an algorithm, which takes an instance of an optimization problem, a parameter $\epsilon>0$ and in polynomial time produces a solution, that is within a factor $1+\epsilon$ of being optimal for minimization problems or $1-\epsilon$ for maximization problems.

We further recall the classes EPTAS (Efficient PTAS), where time complexity is polynomial in the input size (but can have any dependence on $\epsilon$ ) and FPTAS (Fully PTAS), where the time complexity is polynomial in both input size and the parameter $\epsilon$. The class APX contains every problem that can be approximated within a constant factor $c$. 
Previous work $1 D$-SS and $k D$-SS are not strongly NP-complete and can be solved exactly in pseudo-polynomial time: $1 D$-SS is solved in $O(n|t|)$, see [? ]. Generalizing this idea $k D$-SS is solved in $O\left(n|M|^{k}\right)$, where $M=\max P_{n}$ is the farthest reachable point. Moreover, $1 D$-SS is in FPTAS, see [? ]. A related problem is the Multidimensional Knapsack. Firstly, it was proved in $[?]$, that this problem does not have an FPTAS. Later in [? ], does not have an EPTAS, while Knapsack is in FPTAS. $1 D$-SS is a special case of Knapsack as both are defined as maximization problems. In two or higher dimension, it makes more sense to define $k D$-SS as a minimization problem, since a vector sum with maximum length may be far from the target vector. So, in dimension two or higher, the problem is not related to Multidimensional Kanspack but rather to CVP.

A closely connected problem to $k D-\mathrm{SS}$ is the Closest Vector Problem (CVP): we are given a set of basis vectors $B=\left\{b_{1}, \ldots, b_{n}\right\}$, where $b_{i} \in \mathbb{Z}^{k}$, and a target vector $t \in \mathbb{Z}^{k}$, and we ask what is the closest vector to $t$ in the lattice $\mathcal{L}(B)$ generated by $B$. This is $\mathcal{L}(B)=\left\{\sum_{i=1}^{m} a_{i} b_{i} \mid a_{i} \in \mathbb{Z}\right\}$ and thus $k D$-SS is a special case of CVP, where $a_{i} \in\{0,1\}$. CVP is not in APX and cannot even be approximated within a factor of $2^{\log ^{1-\epsilon} n}$ with $\epsilon=(\log (\log n))^{c}$ for $c<1 / 2[?$ ? 1 .

MinkDecomp has its fair share of attention. One application is in the factorization of bivariate polynomials through their Newton polygons. As noticed by Ostrowski in 1921, if a polynomial factors, then its Newton polygon has a Minkowski decomposition. An algorithm for polynomial irreducibility testing using MinkDecomp is presented in [? ] motivated by previous similar work in [? ]. They present a criterion for MinkDecomp that reduces the decision problem into a linear programming question. Continuing the work of $[?$, sections $4,5]$ we propose a polynomial time algorithm, that solves MinkDecomp approximately using a solver for $2 D$-SS. Here, we are interested in finding approximate solutions. We will discuss these problems in ??. MinkDecomp is NP-complete for lattice polygons, and a pseudopolynomial algorithm exists [? ].

Our contribution We introduce the $k D$-SS problem. It is clearly NPcomplete; we prove that it cannot be approximated efficiently. For $k \geq 2$ it cannot be approximated within a constant factor (although the classic 1D-SS has a FPTAS). We design an algorithm for $2 D$-SS-approx: given a set $S,|S|=n$, target $t$ and $0<\epsilon<1$, the algorithm returns, in $O\left(n^{3} \epsilon^{-2}\right)$ time, a subset of $S$ whose vectors sum to $t^{\prime}$ such that $\operatorname{dist}\left(t, t^{\prime}\right) \leq \epsilon M$, where $M=\max P_{n}$. We also describe two more approximation algorithms with a better experimental ratio.

Applying one of these algorithms yields an approximation algorithm for MinkDecomp (??): If $Q$ is the input polygon the algorithm returns polygons $A$ and $B$ whose Minkowski sum defines polygon $Q^{\prime}$ such that $\operatorname{vol}(Q) \leq \operatorname{vol}\left(Q^{\prime}\right) \leq$ $\operatorname{vol}(Q)+\epsilon D^{2}, \operatorname{per}(Q) \leq \operatorname{per}\left(Q^{\prime}\right) \leq \operatorname{per}(Q)+2 \epsilon D, i(Q) \leq i\left(Q^{\prime}\right) \leq \bar{i}(Q)+\epsilon D^{2}$, where $D$ is the diameter of $Q$. The Hausdorff distance of $Q$ and $Q^{\prime}$ is bounded by $d_{H}\left(Q, Q^{\prime}\right) \leq \epsilon / 2 D$.

\section{2. $k D$-SS is not in APX}

To prove that $k D$-SS-opt is not in APX we will apply the idea used to prove that CVP is not in APX, see [? ] for more details. We apply their proof to our problem, in order to prove something similar for the $k D$-SS-opt. 
Proposition 7. /? / For every $c>1$ there is a polynomial time reduction that, given an instance $\phi$ of $S A T$, produces an instance of Set Cover $\left\{\mathcal{U},\left(S_{1}, \ldots, S_{m}\right)\right\}$ where $\mathcal{U}$ is the input set of integers and $S_{1}, \ldots, S_{m}$ are subsets of $\mathcal{U}$, and integer $K$ with the following property: If $\phi$ is satisfiable, there is an exact cover of size $K$, otherwise all set covers have size more than $c K$.

Given a CNF formula $\phi$ we invoke ?? and get an instance of the Set Cover problem. This is a gap introducing reduction, because if $\phi$ is statisfiable then the instance of Set Cover has a solution of size exactly $K$ and if $\phi$ is not statisfiable every solution has size at least $c K$ for a constant $c$. From this instance of Set Cover we create an instance for $k D$-SS that preserves the gap. Now, if $\phi$ is satisfiable, the closest vector to a given target $t$ has distance exactly $K$. If $\phi$ is not statisfiable, the closest vector in target $t$ has distance at least $c K$.

We reduce $k D$-SS to Set Cover for norm $l_{1}$, but this can easily be generalized to any $l_{p}$, where $p$ is a positive integer. We say that a cover is exact if the sets in the cover are pairwise disjoint.

Theorem 8. Given a CNF formula $\phi$ and $c>1$ we create an instance $\left\{v_{1}, \ldots, v_{m} ; t\right\}$ of $k D$-SS. If $\phi$ is satisfiable, then the minimum distance of a possible vector sum from $t$ is smaller than $K$ otherwise, it is larger than $c K$.

Proof. Let $\left\{\mathcal{U},\left(S_{1}, \ldots, S_{m}\right), K\right\}$ be the instance of Set-Cover obtained in Proposition ?? for the formula $\phi$. We transform it to an instance of $k D$-SS with input set $\left\{v_{1}, \ldots, v_{m}\right\}$ and target $t$, such that the distance of $t$ from the nearest point in the set of all possible points $P_{n}$ is either $K$ or $\geq c K$.

Let $v_{i} \in \mathbb{Z}^{n+m}$, where $|\mathcal{U}|=n$. We will create such a vector $v_{i}$ for every set $S_{i} ; 1 \leq i \leq m$. Let $L=c K$. Then the first $n$ coordinates of each vector $v_{i}$ have their $j$ 'th-coordinate $(j \leq n)$ equal to $L$ if the corresponding $j$ 'th-element belongs to set $S_{i}$, or 0 otherwise. The remaining $m$ coordinates have 1 in the $(n+i)^{\prime}$ th-coordinate and zeros everywhere else:

$$
v_{i}=\left(L \cdot \chi_{S_{i}}, 0, \ldots, 1, \ldots 0\right)=\left(L \cdot \chi_{S_{i}}, e_{i}\right),
$$

where $\chi_{S_{i}}$ is the characteristic function of the set $S_{i}$. The target vector $t$ has in the first $n$ coordinates $L$ and the last $m$ coordinates are zeros, $t=$ $(L, \ldots, L, 0, \ldots, 0)$.

Now, let the instance of Set-Cover have an exact cover of size $K$. We will prove that the minimum distance of every $v \in P_{n}$ from target $t$ is less than $K$. Without loss of generality, let the solution be $\left\{S_{1}, \ldots, S_{K}\right\}$. For each $S_{i}, 1 \leq$ $i \leq K$, sum the corresponding vectors $v_{i}$ and let this sum be $\zeta \in \mathbb{Z}^{n+m}$ :

$$
\zeta=\sum_{i=1}^{K} v_{i}=(\underbrace{L, \ldots, L}_{n} \underbrace{1, \ldots, 1}_{K} \underbrace{0, \ldots, 0}_{m-K}) .
$$

The first $n$ coordinates must sum up to $(L, L \ldots, L)$, because if one of the coordinates was 0 , the solution would not be a cover and if one of them was greater than $L$, then some element is covered more than once and the solution would not be exact. Note that each of the first $n$ coordinates is either 0 or greater than $L$. The key point is that in the last $m$ coordinates we will have exactly $K$ units and everything else 0 . The distance of this vector $\zeta$ from $t$ is

$$
\|-t+\zeta\|_{1}=\|(\underbrace{0, \ldots, 0}_{n} \underbrace{1, \ldots, 1}_{K} \underbrace{0, \ldots, 0}_{m-K})\|_{1}=K
$$


Thus, there is a point in $P_{n}$ that its distance from $t$ is at most $K$.

Let us consider the other direction, where the Set Cover instance has a solution set greater than $c K=L$. We will show that the closest vector to $t$ has distance at least $L$ from $t$. This solution must have at least $c K=L$ sets. As before, $\|-t+\zeta\|_{1} \geq L$ (this time the cover need not be exact).

Towards a contradiction, suppose there exists a vector $\xi$ such that $\|-t+$ $\xi \|_{1}<L$. If the corresponding sets do not form a cover of $S$ then one of the first $n$ coordinates of $\xi$ is 0 and this alone is enough for $\|-t+\xi\|_{1}>L$. If the sets form a cover that is not exact, then in at least one of the first $n$ coordinates of $\xi$ will be greater than $L$ (for the element that is covered more than once) and will force $\|-t+\xi\|_{1}$ to be greater than $L$. Finally, if the sets form an exact cover, the first $n$ coordinates of $\|-t+\xi\|_{1}$ will be 0 . For the distance to be less than $L$, in the last $m$ coordinates there must be less than $L$ units implying that the sets in the cover are less than $L$ contradicting our hypothesis.

In all cases, there cannot exist a vector whose distance from $t$ is $<c K$.

Theorem 9. There is no APX for $k D$-SS-approx unless $P=N P$.

Proof. Let $\phi$ be a given formula as an instance of SAT. Use ?? to get an instance of Set Cover and then the reduction from ?? to get an instance of $k D$-SS. Suppose there exists an algorithm $\mathcal{A}$ for $k D$-SS-opt that is in APX. $\mathcal{A}$ returns a vector $t^{\prime}$ such that $\left\|t-t^{\prime}\right\|_{1} \leq(1+\epsilon) O P T$, where $O P T=\left\|t-t^{*}\right\|_{1}$ and $t^{*}$ is the closest vector in $P_{n}$. From ??, if $\phi$ is satisfiable then $O P T \leq K$ and if $\phi$ is not satisfiable $O P T>c K$.

We must run algorithm $\mathcal{A}$ with a suitable parameter $\epsilon$ so we can distinguish if the optimum solution $t^{*}$ is within distance $K$ or not. When $\phi$ is satisfiable we would want $(1+\epsilon) K<c K \Longrightarrow \epsilon<c-1$. Set $c^{\prime}<c-1$, call $\mathcal{A}$ with parameter $\epsilon=c^{\prime}$ and let $t^{\prime}$ be the returned vector. In the case where $\phi$ is satisfiable and $O P T \leq K$ we have

$$
\left\|t-t^{\prime}\right\|_{1} \leq(1+\epsilon) O P T<c K
$$

Of course if $\phi$ is not satisfiable for any $t^{\prime}$ we have that $\left\|t-t^{\prime}\right\|_{1}>c K$. Thus, $\left\|t-t^{\prime}\right\|_{1}<c K$ if and only if $\phi$ is satisfiable. Since $\epsilon$ is a constant and $\mathcal{A}$ is in APX we can decide SAT in polynomial time.

Although there can be no algorithm that returns a constant factor approximation solution for general dimension $k$, we will present algorithms that provide different kind of approximation. Specifically, the returned vector $t^{\prime}$ of our algorithm is an $(O P T+\epsilon M)$ solution, where $M=\max P_{n}$ is the longest possible vector sum.

\section{Three approximation algorithms for $2 D$-SS}

\section{1. annulus-slice algorithm}

The idea here is to create all possible vectors step by step. At each step, if two vectors are close to each other, one is deleted. Whenever we refer to distance it is the Euclidean distance. We begin with some notation.

- Input: the set $S=\left\{v_{1}, v_{2}, \ldots, v_{n}\right\}$ with $v_{i}=\left(x_{i}, y_{i}\right) \in \mathbb{Z}^{2}$ and $|S|=n$, parameter $0<\epsilon<1$. 

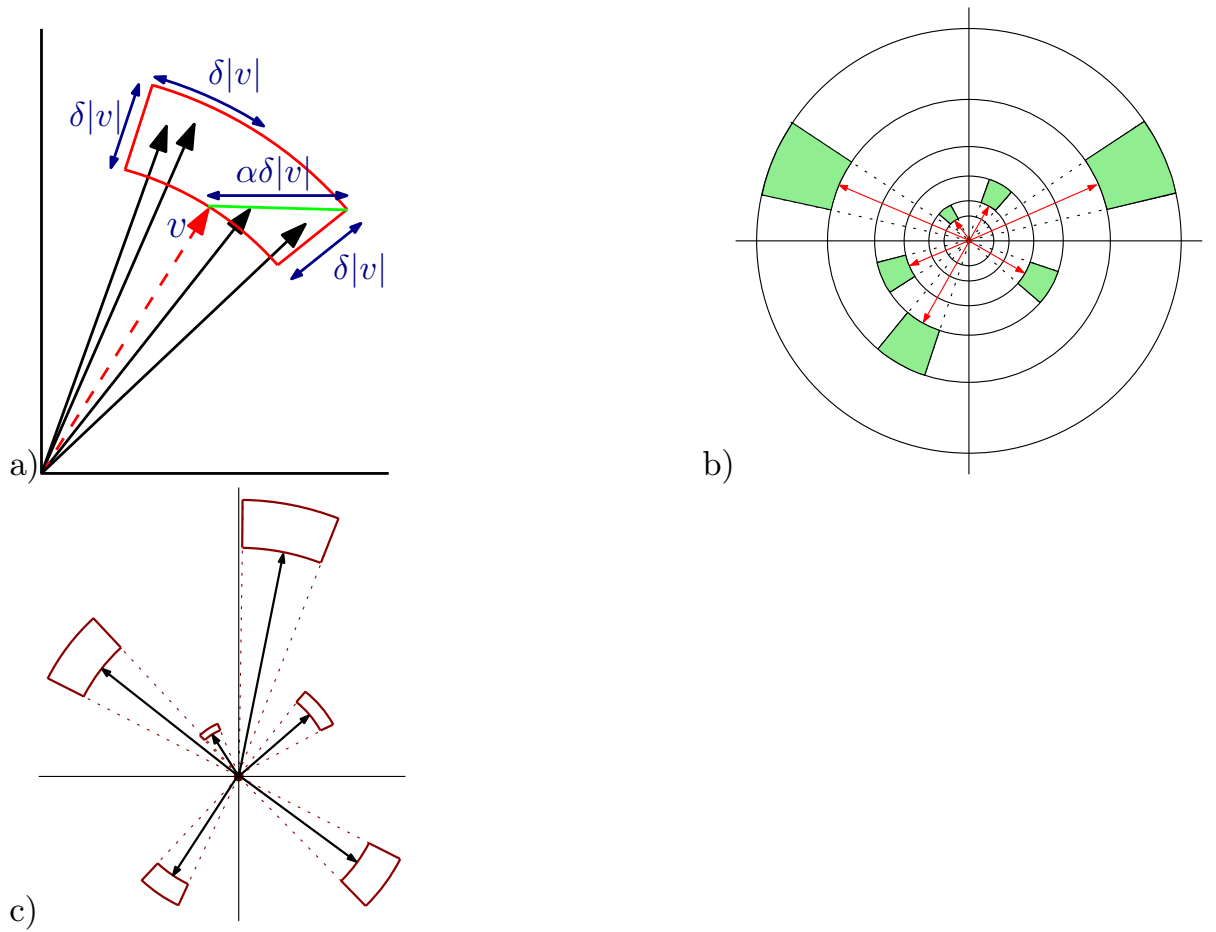

Figure 1: a) A single cell for the dashed vector $v$. All vectors in the cell will be deleted. The distances are shown and the furthest point is in distance $\alpha \delta|v|$. b) How space is divided. c) A few cells. The shorter the vector the smaller the cell.

- $P_{i}$ is the set of all possible vectors that can be produced by adding at most $i$ elements from the first $i$ vectors in $S . P_{n}$ is the set of all possible vector sums.

- $E_{i}=L_{i-1} \cup\left\{w+v_{i} \mid w \in L_{i-1}\right\}$ is the list created at the beginning of every step and that is about to get trimmed.

- $L_{i}=\operatorname{trim}\left(E_{i}, \delta\right)$ is the trimmed list and $0 \leq \delta \leq 1$.

At the beginning of the $i$-th step we create the list $E_{i}=L_{i-1} \cup\left\{w+v_{i} \mid w \in\right.$ $\left.L_{i-1}\right\}$. Notice that addition is over $\mathbb{Z}^{2}$. After a point is found we calculate its length, sort $E_{i}$ based on the lengths and call $\operatorname{trim}\left(E_{i}, \epsilon / 2 n\right)$. For each vector $u \in E_{i}$ with length $|u|$ and angle $\theta(u)$ from the $x$-axis, check all the vectors $u^{\prime} \in E_{i}$ that have length $|u| \leq\left|u^{\prime}\right| \leq(1+\delta)|u|$. If also $\theta(u)-\delta \leq \theta\left(u^{\prime}\right) \leq \theta(u)+\delta$, remove $u^{\prime}$ from $E_{i}$. The remaining trimmed list is the list $L_{i}$. The two conditions ensure that $\operatorname{dist}\left(u^{\prime}, u\right) \leq \alpha \delta|u|$, where $1 \leq \alpha \leq 2$ is a constant. Every vector that is deleted from $E_{i}$ is not very far away from a vector in $L_{i}$ :

$$
\forall u \in E_{i}, \exists w \in L_{i}: u=w+r_{w},\left|r_{w}\right| \leq \alpha \delta|w|
$$

hence, $|w| \leq|u| \leq(1+\delta)|w|$. See ??.

Since all vectors have integer coordinates, any vector $u \in E_{i}$ such that $|u|<1 / \alpha \delta<\sqrt{2} n / \epsilon$ implies that $\alpha \delta|u|<1$. Thus, the area around $u$ does not contain any other other lattice points except $u$. 
Lemma 10. Using the above notation, call function $L_{i}=\operatorname{trim}\left(E_{i}, \delta\right)$, with parameter $\delta=\epsilon / 2 n$ and let $M_{i}=\max \left\{|u|: u \in E_{i}\right\}$, the vector in $E_{i}$ with the largest length. It holds that $\left|L_{i}\right|=O\left(n^{2} \epsilon^{-2} \log M_{n}\right)$ for $1 \leq i \leq n$.

Proof. Every vector in $E_{i}$ has length between $(1+\delta)^{k}$ and $(1+\delta)^{k+1}$. These are circles with center $(0,0)$ and radius $(1+\delta),(1+\delta)^{2}, \ldots,(1+\delta)^{k}$ for some $k$. Every two successive circles form an annulus that we call it a zone. We must cover all $u \in P_{n}$ and $k$ is the minimum such that $(1+\delta)^{k}>M_{n}$. Solving $(1+\delta)^{k} \geq M_{n}$ for $k$, there are $O\left(n \log M_{n} / \epsilon\right)=O\left(n^{2} / \epsilon\right)$ many zones that can be created.

Every zone is divided into cells. Each cell is taken in such a way that it will cover $2 \delta R$ of the inner circle of the zone, where $R$ is the radius of this circle (??a). Thus, every zone between the circles with radious $R$ and $(1+\delta) R$ has at most $2 \pi R / \delta R=4 \pi n / \epsilon$ cells.

Since a list $L_{i}$ has at most an entry for every cell created in every zone, its size can be at most $\left(n^{2} / \epsilon\right) \cdot(4 \pi n / \epsilon)=O\left(n^{3} \epsilon^{-2}\right)$.

For function trim, the time required is $\left|E_{i}\right|$ to consider all vectors and, in the worst case, we have to check each vector in $E_{i}$ with all the others leading to a running time of $O\left(\left|E_{i}\right|^{2}\right)=O\left(\left|L_{i}\right|^{2}\right)$. The running time for ?? is $n$. $T($ trim $)=O\left(n\left|L_{n}\right|^{2}\right)$ and overall, from ??, it requires time $O\left(n^{5} \epsilon^{-4} \log ^{2} M_{n}\right)$. The algorithm only stores at each step the list $L_{i}$ so the space consumption is $O\left(n^{2} \epsilon^{-2} \log M_{n}\right)$.

Corollary 11. For $\delta=\epsilon / 2 n$ the running time of ?? is $O\left(n^{5} \epsilon^{-4} \log ^{2} M_{n}\right)$ and space required is $O\left(n^{2} \epsilon^{-2} \log M_{n}\right)$.

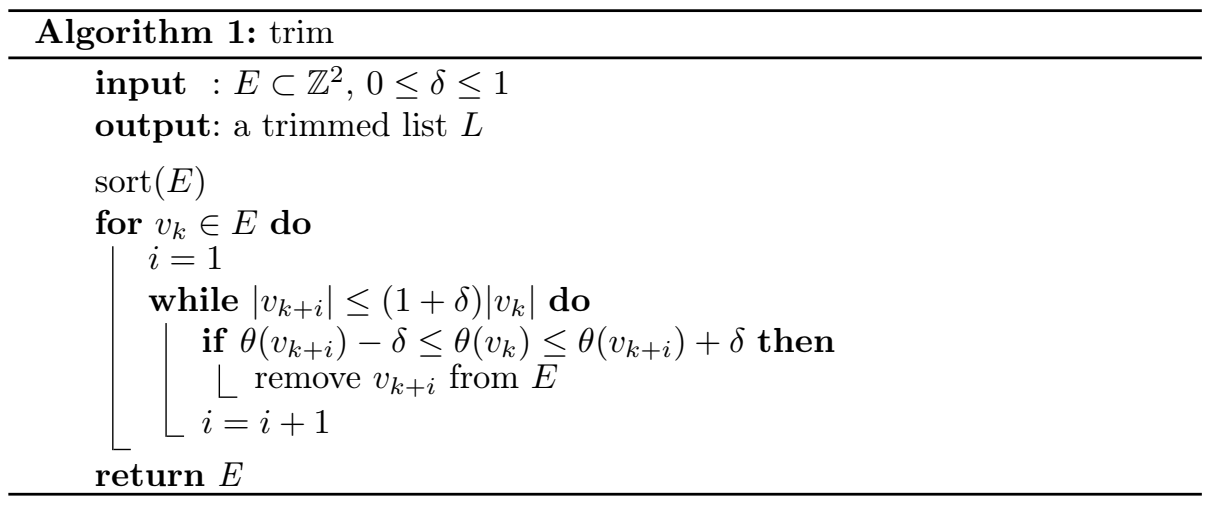

Theorem 12. For a set of vectors $S=\left\{v_{i} \mid v_{i} \in \mathbb{Z}^{2}, 0 \leq i \leq n\right\}$, every possible vector sum $v \in P_{n}$ can be approximated by a vector $w$ such that

$$
\forall v \in P_{n}, \exists w \in L_{n}, \exists r_{w} \in \mathbb{Z}^{2}: v=w+r_{w},\left|r_{w}\right| \leq n \delta M_{n}
$$

Proof. The proof is by induction. The base step, it is easy to see that if we only have one element the theorem holds. The induction hypothesis

$$
\forall v \in P_{n-1}, \exists w \in L_{n-1}, \exists r_{w}: v=w+r_{w},\left|r_{w}\right| \leq(n-1) \delta M_{n-1} .
$$




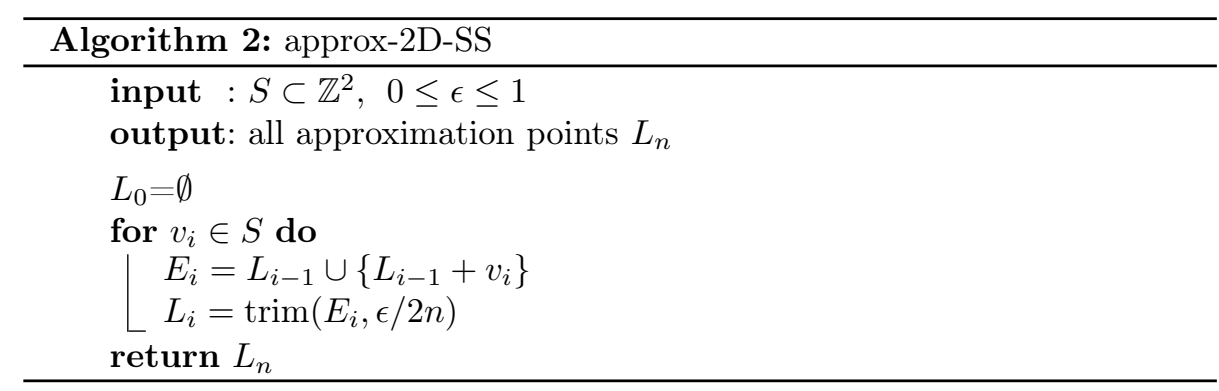

Now suppose $v \in P_{n} \backslash P_{n-1}$, because if $v \in P_{n-1}$ the theorem holds straight from the induction hypothesis. We write $v$ as $v=z+v_{n}, z \in P_{n-1}$ and the induction hypothesis holds for $z$, thus

$$
\exists p \in L_{n-1}, \exists r_{p}: z=p+r_{p},\left|r_{p}\right| \leq(n-1) \delta M_{n-1} .
$$

Since $p \in L_{n-1}$ this means that $p+v_{n} \in E_{n}$ and $L_{n}=\operatorname{trim}\left(E_{n}\right)$. From the guarantee of function trim we know that

$$
\exists q \in L_{n}: p+v_{n}=q+r_{q},\left|r_{q}\right| \leq \delta|q| .
$$

From ???? we get $v=z+v_{n}=p+v_{n}+r_{p}=q+r_{q}+r_{p}$. This proves that for $v \in P_{n}$ there exists a vector $q \in L_{n}$ that approximates it; but how close are they? We will bound the length $\left|r_{q}+r_{p}\right|$. From ????,

$$
\begin{aligned}
& \left|r_{p}\right| \leq(n-1) \delta \max \left\{L_{n-1}\right\} \leq(n-1) \delta M_{n} \\
& \left|r_{q}\right| \leq \delta|q|, q \in L_{n} \Longrightarrow\left|r_{q}\right| \leq \delta M_{n}
\end{aligned}
$$

Thus

$$
\left|r_{q}+r_{p}\right| \leq\left|r_{q}\right|+\left|r_{p}\right| \leq(n-1) \delta M_{n}+\delta M_{n} \leq n \delta M_{n} .
$$

Setting $\delta=\epsilon / 2 n$ we can ensure that every possible vector sum will be approximated by a vector in $L_{n}$ at most $\epsilon M_{n}$ far. Implementing and testing the algorithm, much better bounds are obtained, see ??.

\subsection{A grid based algorithm}

Our input is a list of vector $S=\left\{v_{i} \mid v_{i} \in \mathbb{Z}^{2}, i \leq n\right\}$. We define the list $E_{i}=L_{i-1} \cup\left\{w+v_{i} \mid w \in L_{i-1}\right\}$ and at each step $i$ we trim it by a parameter $\delta$ to get the trimmed list $L_{i}=\operatorname{trim}\left(E_{i}, \delta\right)$. Also, $P_{i}$ is the set of all possible vector sums using a subset of the first $i$ vectors of $S, P_{i}=\left\{\sum_{j}^{i} a_{j} v_{j} \mid a_{j} \in\{0,1\}, v_{j} \in\right.$ $S\}$.

It turns out that the same approximation ratio $\epsilon M_{n}$ can be achieved by a faster algorithm that separates the plane into a grid, where $M_{n}$ is the length of the largest vector in $E_{i}$. Instead of creating this different annulus-slice cells we have a regular orthogonal grid where each square cell has side $d=\epsilon M_{n} / 2 n$. Many thanks to Günter Rote for the fruitful conversation.

Let the cell side length be $d=\epsilon M_{n} / 2 n$, and for each $v(x, y) \in E_{i}$ store in the trimmed list $L_{i}$ the vector with its coordinates rounded in the integer multiple of $d$ :

$$
\forall v(x, y) \in E_{i} \exists w\left(x^{\prime}, y^{\prime}\right) \in L_{i}: x^{\prime}=\left\lfloor\frac{x}{d}\right\rfloor, y^{\prime}=\left\lfloor\frac{y}{d}\right\rfloor
$$




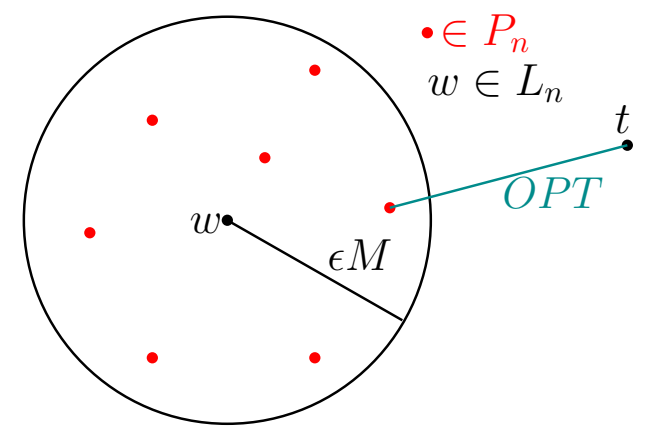

Figure 2: For every $t$ the returned vector is at most $\epsilon M$ from the optimum

and

$$
\operatorname{dist}(v, w) \leq \sqrt{2} d \leq \frac{\epsilon M_{n}}{n}
$$

and the maximum value reaches when $v$ and $w$ are in the diagonal of the cell.

The whole grid has size $2 M_{n}$ and since $d=\epsilon M_{n} / 2 n$ the grid has $O\left(\left(M_{n} / d\right)^{2}\right)=$ $O\left((n / \epsilon)^{2}\right)$ cells. In the worst case we will have a vector in every cell and this means that the time to traverse the lists $E_{i}$ at each step is $O\left(n^{2} \epsilon^{-2}\right)$. Since we have $n$ lists the total running time of the new algorithm is $O\left(n^{3} \epsilon^{-2}\right)$ and the space requirements are $O\left(n^{2} \epsilon^{-2}\right)$.

Also, every $u \in P_{n}$ is the sum of at most $n$ vectors from $S$. In the worst case, every time we call trim, we represent a vector $u \in E_{i}$ by another one that has distance form $u$ at most $d$. In that case we lost at most $n d=\epsilon M_{n}$ :

$$
\forall v \in P_{n} \exists w \in L_{n}: \operatorname{dist}(v, w) \leq \epsilon M_{n} .
$$

Thus, for every given target vector $t$ the algorithm will return an approximation solution that is $n d=\epsilon M_{n}$ far from being optimum ??.

Corollary 13. The grid-based algorithm runs in time $O\left(n^{3} \epsilon^{-2}\right)$, requires space $O\left(n^{2} \epsilon^{-2}\right)$ and returns a solution $t^{\prime}$ such that dist $\left(t, t^{\prime}\right) \leq O P T+\epsilon M_{n}$

In $2 \mathrm{D}$ case there is a factor $\sqrt{2}$ that we also omit it from our approximation. This happens because the maximum distance inside a cell is not $d$ but $\sqrt{2} d$. For dimension $k$ the minimum distance is $\sqrt{k} d$ and this affects the algorithm in higher dimension.

\subsection{A heuristic method}

The grid-based algorithm "cuts" a constant d from every factor regardless of its length. If a vector has length $10,10^{3}$ or $10^{5}$, the algorithm will behave the same. On the other hand, it is fast, because it does not have to check any other vector; for every vector it sees it rounds it on the spot, thus having linear time in the size of the lists. We can make a version of a circular grid, where each cell does not have a constant side length. For small vectors we create smaller cells and as the length increases so does the cell side. This way we can provide a better experimental approximation ratio. At step $i$ we have the list $E_{i}$ and we will trim it with a factor $\delta$. We will consider the polar coordinates of the vectors. For a vector $v(\phi, r)$ let $\phi=\theta(v)$ be the angle with the $x$ axis and $r=|v|$ its 
euclidean length. Let $v$ be a vector in $E_{i}$ and, to get the list $L_{i}$, we will replace $v$ by $v^{\prime}=\left(\phi^{\prime}, r^{\prime}\right)$. First round its angle in multiple of $\delta: \phi^{\prime}=\lfloor\phi / \delta\rfloor$. Next, we round its length. The idea is to round in such a way that shorter vectors are approximated better than the longer ones. Rounding to a multiple of some $d$ does not provide that. For rounding the lengths we will construct an array $A$ with all the acceptable rounded lengths. The entries of $A$ are the lengths $\left[1,(1+\delta), \ldots,(1+\delta)^{i}\right]$ for the minimum $i$ such that $(1+\delta)^{i}>M_{n}$. Solving this inequality we get that $i=O\left(n \log M_{n} / \epsilon\right)$ and this is the size of $A$. Now, for a vector $v$ we just make a binary search in $A$ for $|v|$ that returns the zone such that $(1+\delta)^{k} \leq|v| \leq(1+\delta)^{k+1}$ and $r^{\prime}=b i n \_\operatorname{search}(A,|v|)=(1+\delta)^{k}$. The space is divided in $O(\delta)=O(n / \epsilon)$ angles and $O\left(n \log M_{n} / \epsilon\right)$ different lengths. Each $E_{i}$ has size $O\left(n^{2} \epsilon^{-2} \log M_{n}\right)$; we save the quadratic factor, but add a $\log \left|E_{i}\right|$ for the binary search. The whole algorithm runs in time

$$
O\left(n\left|E_{i}\right| \log \left|E_{i}\right|\right)=O\left(n^{3} \epsilon^{-2} \log M_{n} \log \frac{n^{2} \log M_{n}}{\epsilon^{2}}\right)
$$

and provides the same approximation, since $\forall v \in E_{i}, \exists w \in \operatorname{Li}: \operatorname{dist}(v, w) \leq \epsilon|w|$ as before. We believe that the better behaviour of this algorithm can be suited for an average case analysis, that will prove that the algorithm provides a better approximation ratio for the majority of $v \in P_{n}$. Also, the binary search may be dropped by using a method to round the lengths in $O(1)$ time, dropping this way the $\log \left|E_{i}\right|$ factor.

\section{Minkowski Decomposition using 2D-SS}

In this section, we will describe an algorithm for approximating MinkDecomp. The algorithm takes an input polygon $Q$, transforms it to an instance $\{S, t\}$ of $2 D$-SS-approx and calls the algorithm for $2 D$-SS-approx. Then it takes the output and converts it to an approximate solution to MinkDecomp.

Let $Q$ be the input to MinkDecomp: $Q=\left\{v_{i} \mid v_{i} \in \mathbb{Z}^{2}, 0 \leq i \leq n\right\}$, such that $\sum_{1}^{n} v_{i}=(0,0)$. First, we create the vector set $s(Q)$ by subtracting successive vertices of $Q$ (in clockwise order): $s(Q)=\left\{v_{0}-v_{1}, v_{1}-v_{2}, \ldots, v_{n}-v_{0}\right\}$. Each vector in $s(Q)$ is called an edge vector and $s(Q)$ is called the edge sequence of $Q$. For each edge vector in $s(Q)$ we calculate its primitive vector.

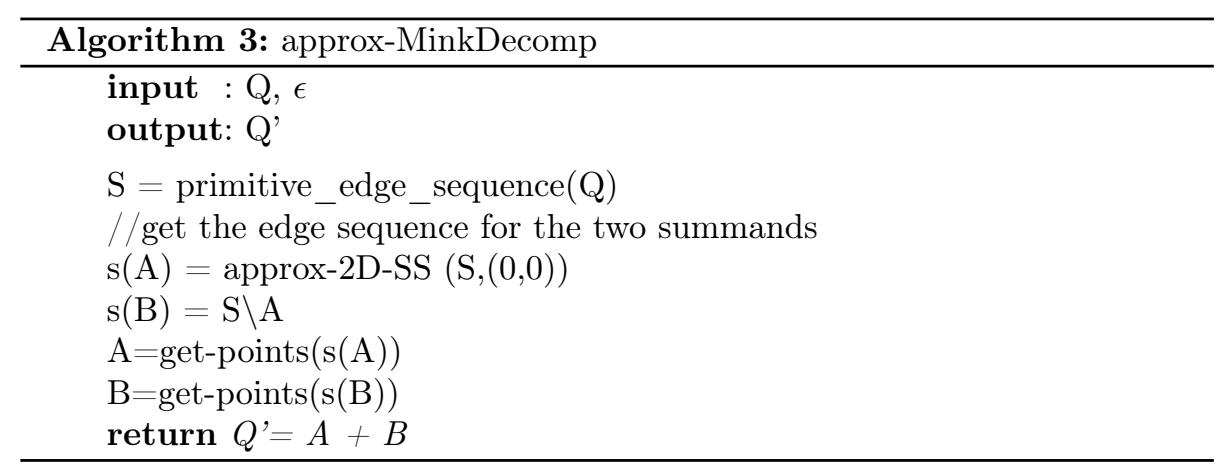

Definition 14. Let $v=(a, b)$ be a vector and $d=\operatorname{gcd}(a, b)$. The primitive vector of $v$ is $e=(a / d, b / d)$. 


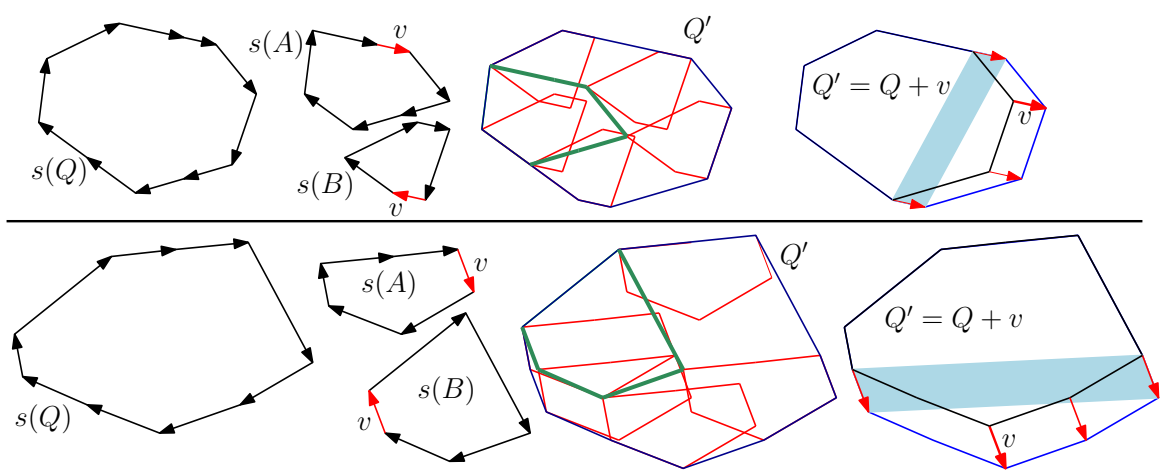

Figure 3: Two examples for two polygons $Q$. Their summands are shown and the red vector $v$ is the new vector added to fill the gap. At the end, the new polygon $Q^{\prime}$ is Minkowski Sum of the two summands.

We get an edge vector $(x, y) \in s(Q)$ and calculate its primitive vector $e=$ $(x / d, y / d)$, where $d=\operatorname{gcd}(x, y)$. We could create the set $S$ by adding in it $d$ times the vector $e$ for every $v \in s(Q)$ but this may create a set $S$ that has exponential size to the edges of the polygon. Instead, we computer the scalars $d_{1}, \ldots, d_{k}$ by the following formulas

$$
d_{i}=2^{i}, i=0, \ldots,\left\lfloor\log _{2} d / 2\right\rfloor \text { and } d_{k}=d-\sum_{i=1}^{\left\lfloor\log _{2} d / 2\right\rfloor} d_{i}
$$

We create the set $S$ from the vectors $d_{i} e$ and repeat the procedure for all vectors $v \in s(Q)$. Notice that $\sum_{1}^{k} d_{i}=d$, so the primitive edge sequence also sums to $(0,0)$. Using this construction, the primitive vectors added are at most $\log d$ for every $v \in s(Q)$ keeping the size of $S$ polynomial with respect to the edges of $Q$. The primitive edge sequence uniquely identifies the polygon up to translation determined by $v_{0}$. This is a standard procedure as in [? ? ].

The main defect in this approach is that the algorithm returns a sequence of vectors $S^{\prime} \subset S$ that sum close to $(0,0)$ but possibly not $(0,0)$. This means the corresponding edge sequence does not form a closed polygon. To overcome this, we just add to $s(A)$ the vector $v$, from the last point to the first, to close the gap. If $s(A)$ sums to a point $(a, b)$, by adding vector $v=(-a,-b)$ to $s(A)$ the edge sequence $s(A) \cup\{v\}$ now sums to $(0,0)$. If we rearrange the vectors by their angles, they form a closed, convex polygon that is summand $A$. We do the same for the sequence $s(B)$. The vector added in $s(B)$ is $-v=(a, b)$ and this sequence (rearranged) also forms a closed, convex polygon. We name $s\left(A^{\prime}\right)=s(A) \cup\{v\}$, $s\left(B^{\prime}\right)=s(B) \cup\{v\}$ and take their Minkowski Sum $Q^{\prime}=A^{\prime}+B^{\prime}$, where $A^{\prime}$ and $B^{\prime}$ are the convex polygons formed by $s\left(A^{\prime}\right)$ and $s\left(B^{\prime}\right)$. We measure how close $Q^{\prime}$ is to the input $Q$. Let $D$ be the diameter of $Q$, the maximum distance between two vertices of $Q$.

Lemma 15. Let $Q$ be the input polygon and $Q^{\prime}$ be the polygon as discussed above. vol stands for volume. per for perimeter, $i$ are the interior lattice points of a polygon and $d_{H}$ the Huasdorff distance. We deduce that

1. $\operatorname{vol}(Q) \leq \operatorname{vol}\left(Q^{\prime}\right) \leq \operatorname{vol}(Q)+\epsilon D^{2}$

2. $\operatorname{per}(Q) \leq \operatorname{per}\left(Q^{\prime}\right) \leq \operatorname{per}(Q)+2 \epsilon D$ 


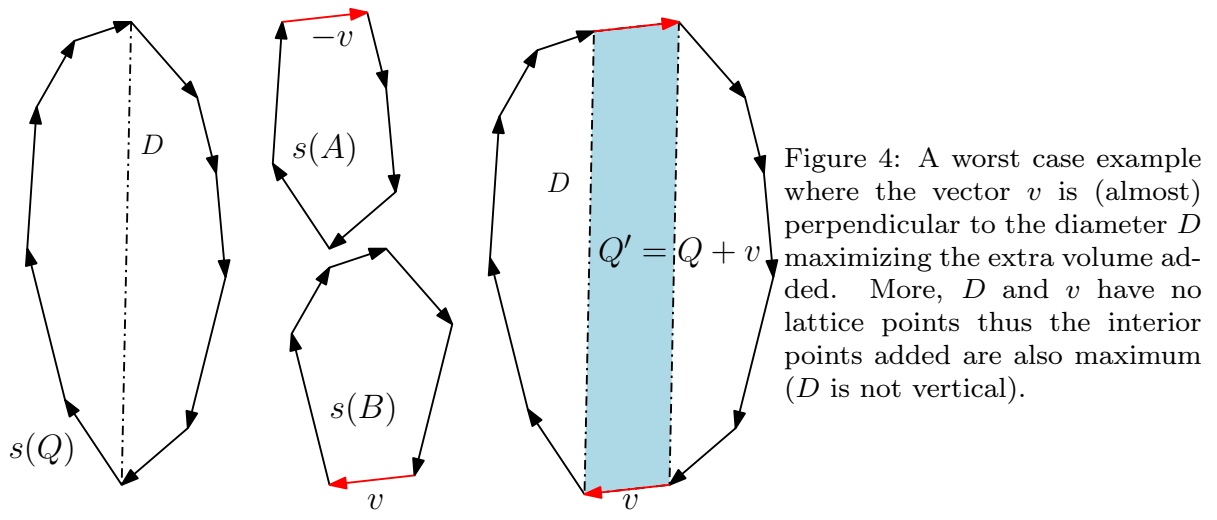

3. $i(Q) \leq i\left(Q^{\prime}\right) \leq i(Q)+\epsilon D^{2}$

4. $d_{H}\left(Q, Q^{\prime}\right) \leq \epsilon / 2 D$

Proof. We observe that

$$
\begin{aligned}
& s\left(Q^{\prime}\right)=s\left(A^{\prime}\right) \cup s\left(B^{\prime}\right)=s(A) \cup s(B) \cup\{v\} \cup\{\} \cup\{-v\} \Longrightarrow \\
& s\left(Q^{\prime}\right)=s(Q) \cup\{v\} \cup\{\} \cup\{-v\} .
\end{aligned}
$$

This equals adding to $Q$ a single segment $s$ of length $|s|=|v|$ and $Q^{\prime}=Q+s$. The length of vector $v$ we add to close the gap, is the key factor to bound polygon $Q^{\prime}$. From the guarantee of the $2 D$-SS-approx algorithm we know that $s(A)$ (and respectively $s(B))$ sum to a vector with length at most $\epsilon \max \left\{L_{n}\right\}$. This is vector $v$ and thus $|v| \leq \epsilon \max \left\{L_{n}\right\}$. Since $\max \left\{L_{n}\right\} \leq D$, we get $|s|=|v| \leq \epsilon D$.

From above we easily see that:

1. $\operatorname{per}(Q)=\sum_{v \in s(Q)}|v|$, it follows $\operatorname{per}\left(Q^{\prime}\right)=\operatorname{per}(Q)+2|v| \leq \operatorname{per}(Q)+2 \epsilon D$.

2. $\operatorname{vol}\left(Q^{\prime}\right) \leq \operatorname{vol}(Q)+s D \leq \operatorname{vol}(Q)+\epsilon D^{2}$

3. By Pick's theorem, $\operatorname{vol}(Q)=i(Q)+b(Q) / 2-1 \Longrightarrow i(Q)=\operatorname{vol}(Q)-$ $b(Q) / 2+1$. Note that $b(Q)=\sum_{\forall v \in s(Q)} d_{v}$ where $v=(x, y) \in s(Q)$ and $d_{v}=\operatorname{gcd}(x, y)$ as is ??. Now, $i\left(Q^{\prime}\right)=i(Q)+i(s D)$ since $s D$ is the maximum volume added and $i(s D) \leq s D-b(s D) / 2+1 \leq s D-1 \leq \epsilon D^{2}$. Thus, $i\left(Q^{\prime}\right) \leq i(Q)+\epsilon D^{2}$

4. If we "slide" $Q$ by $s / 2$ units in the direction of $v, d_{H}\left(Q, Q^{\prime}\right)=s / 2 \Longrightarrow$ $d_{H}\left(Q, Q^{\prime}\right) \leq \epsilon / 2 D$

A bad example can be seen in ??, where the added vector is (almost) perpendicular to $D$ maximizing the extra volume and internal lattice points. ?? leads to following conclusion:

Corollary 16. The proposed algorithm provides a $2 \epsilon D$-solution for MinkDecompper-approx, a $\epsilon D^{2}$-solution for MinkDecomp-vol-approx and MinkDecomp-latt_papprox and a $\epsilon / 2 D$-solution for MinkDecomp-d $d_{H}$-approx. 
a)

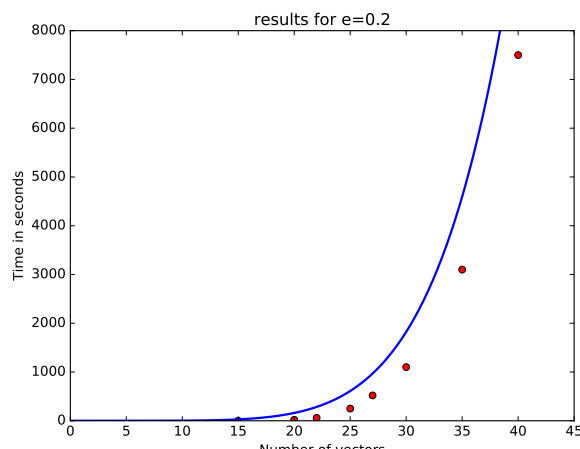

c)

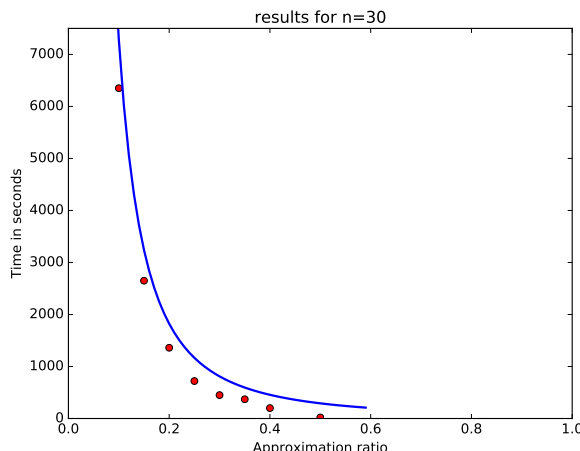

b)

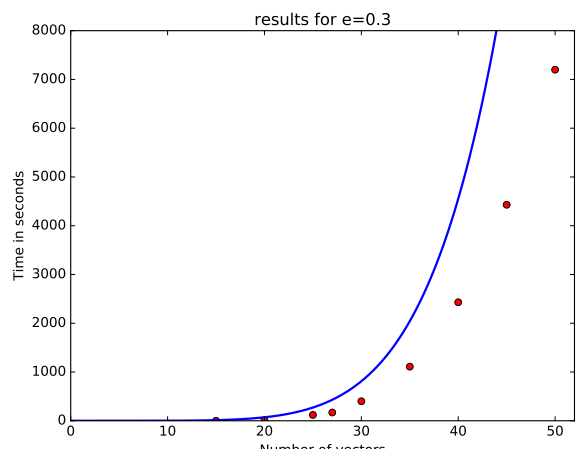

d)

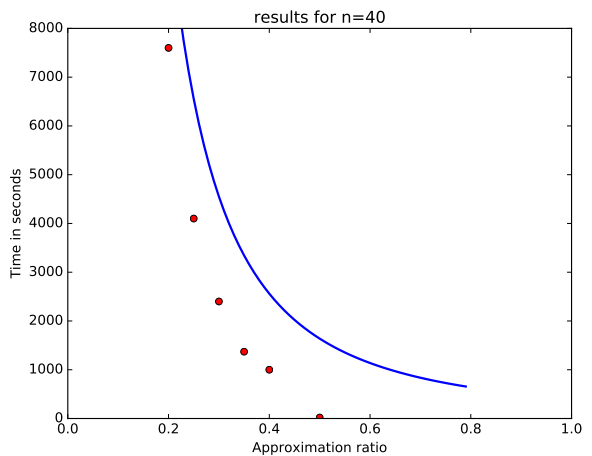

Figure 5: Experimental results for 2D-SS-approx: a) $\epsilon=0.2$ and b) $\epsilon=0.30$, c) $n=30$ and d) $n=40$. The blue line is the expected time, the red dots our experiments.

\section{Implementation and experimental results.}

We implement both ???? in Python3. The code can be accessed through Github ${ }^{1}$ and is roughly 750 lines long. We provide methods for either $2 D$-SSapprox or MinkDecomp- $\mu$-approx. To test ?? we created vectors $v_{i}$ at random with $\left|v_{i}\right| \leq 5000$. For ?? we create random points and take their convex hull to form input polygon $Q$. All tests were executed in an Intel Core i5-2320@3.00 GHz with 8Gb RAM, 64-bit Ubuntu GNU/Linux. Results for ?? are shown in ?? and for algorithm ?? in ??. It is clear in ?? that our results stay below the expected time and behave analogously. In ?? the results obtained are much better than the proven bounds and in most cases volume and perimeter are almost the same and the polygons differ slightly.

\section{Further work}

In this work we presented some theoretical results concerning the approximation of two NP-hard problems: $k D$-SS and Minkdecomp. Additionally, we provide polynomial time approximation algorithms to solve these problems. The

\footnotetext{
${ }^{1}$ https://github.com/tzovas/Approximation-Subset-Sum-and-Minkowski-Decomposition
} 


\begin{tabular}{|c|c|c|c|c|c|c|}
\hline \#vertices & \# examples & $\operatorname{vol}(\mathrm{Q}) / \operatorname{vol}\left(\mathrm{Q}^{\prime}\right)$ & $\operatorname{per}(\mathrm{Q})-\operatorname{per}\left(\mathrm{Q}^{\prime}\right)$ & Hausdorff & $\epsilon$ & time(secs) \\
\hline 3âĂ & $\overline{51}$ & "0,93 & 18,55 & $3 \overline{3,32}$ & (0,18 & $4 \overline{4,1}$ \\
\hline 11âAT 16 & 45 & 0,977 & 3,43 & 1,81 & 0,33 & 126,4 \\
\hline 17âẢ 25 & 54 & 0,994 & 1,12 & 1,25 & 0,38 & 377,5 \\
\hline
\end{tabular}

Table 1: Input polygon $Q$, output $Q^{\prime}(\operatorname{per}(Q)>1000)$. Gather examples by the number of their vertices. We measure volume, perimeter and Hausdorff distance and present their mean values.

next step is to associate these ideas with certain algebraic problems like approximate polynomial factoring or irreducibility testing.

Given a polynomial $f_{Q}$ and its Newton polygon $Q$, we use the MimkDecomp approximation algorithm to find an approximation decomposition $Q^{\prime}=A^{\prime}+B^{\prime}$. Using the irreducibility test in [? ], we either find a binary bivariate factorization or that $f_{Q^{\prime}}$ is irreducible. In the second case, we use the approximate polynomial factorization algorithm in [?]. All monomials of $f_{Q}$ lie in the support of $f_{Q}^{\prime}$ therefore the corresponding coefficients should be same with the coefficients of these monomials in $f_{Q}^{\prime}$. The new terms in $f_{Q}^{\prime}$ have coefficients, whose value is to be determined In other words, we need to determine, whether there exist valid coefficients for the monomials that correspond to lattice points in $Q^{\prime} \backslash Q$. 